\title{
Persistent distress after psychological exposure to the Nagasaki atomic bomb explosion
}

\author{
Yoshiharu Kim, Atsuro Tsutsumi, Takashi Izutsu, Noriyuki Kawamura, Takao Miyazaki
}

and Takehiko Kikkawa

\section{Background}

Although there is speculation that individuals living in the vicinity of nuclear disasters have persistent mental health deterioration due to psychological stress, few attempts have been made to examine this issue.

\begin{abstract}
Aims
To determine whether having been in the vicinity of the Nagasaki atomic bomb explosion in the absence of substantial exposure to radiation affected the mental health of local inhabitants more than half a century later.

\section{Method}

Participants were randomly recruited from individuals who lived in the vicinity of the atomic bomb explosion in uncontaminated suburbs of Nagasaki. This sample $(n=347)$ was stratified by gender, age, perception of the explosion and current district of residence. Controls $(n=288)$ were recruited from among individuals who had moved into the area from outside Nagasaki 5-15 years after the bombing, matched for gender, age and district of residence. The primary outcome measure was the proportion of those at high risk of mental disorder based on the 28 -item version of the General Health Questionnaire, with a cut-off point of 5/6. Other parameters related to individual perception of the
\end{abstract}

explosion, health status, life events and habits were also assessed.

\section{Results}

Having been in the vicinity of the explosion was the most significant factor $(\mathrm{OR}=5.26,95 \% \mathrm{Cl} 2.56-11.11)$ contributing to poorer mental health; erroneous knowledge of radiological hazard showed a mild association. In the sample group, anxiety after learning of the potential radiological hazard was significantly correlated with poor mental health $(P<0.05)$, whereas anxiety about the explosion, or the degree of perception of it, was not; $74.5 \%$ of the sample group believed erroneously that the flash of the explosion was synonymous with radiation.

\section{Conclusions}

Having been in the vicinity of the atomic bomb explosion without radiological exposure continued to be associated with poorer mental health more than half a century after the event. Fear on learning about the potential radiological hazard and lack of knowledge about radiological risk are responsible for this association.

\section{Declaration of interest}

None.
Persistent mental health problems among local inhabitants and people entering the affected area after nuclear disasters has been repeatedly reported. ${ }^{1-11}$ It has been suggested that non-radiological factors such as traumatic fear, secondary distress, fear of invisible or latent radiological harm, social turmoil or isolation might be responsible., ${ }^{3,4,9,12}$ This view accords with current knowledge of radiology, as postnatal radiation effects on the brain have been documented after radiation therapy for a brain tumour in which fractionated doses above a total of $40 \mathrm{~Gy}$ were received, ${ }^{4}$ whereas the estimated $\mathrm{LD}_{50 / 60}$ for survivors of the atomic bomb has been reported not to have exceeded $3 \mathrm{~Gy}$, as calculated in Hiroshima. ${ }^{13}$ Although the participants in previous studies conducted after the nuclear events at Hiroshima, Nagasaki and Chernobyl had mostly experienced some degree of radiation exposure, it has not been demonstrated whether, or to what extent, certain types of non-radiological factors cause persistent mental health deterioration through comparison with adequate controls. Studies of non- exposed but psychosociologically involved residents were conducted after the accident at the Three Mile Island nuclear plant, ${ }^{14}$ but the last observation was made only a few years after the event and was based on a small, non-randomised group, ${ }^{15}$ thus providing somewhat insubstantial information. Our intention in this study, therefore, was specifically to delineate the persistent mental effects of a nuclear disaster in the absence of substantial radiological health effects. To our knowledge no such study has been reported previously.

\section{Method}

\section{Participants}

The sample group was recruited from among individuals who had lived in the peripheral areas of Nagasaki since the atomic bomb explosion in 1945 until the time of the study; these districts were Mogi, Himi, Higashi-Nagasaki, Shikimi, Mie, Fukabori, Ioujima, Tokitsu, Kinkai, Tarami and Iimori, all adjacent to the inner contaminated zone. Although these individuals had been in the vicinity of the explosion the areas in question had been officially recognised as uncontaminated, and in 1994 it was confirmed by a government research team that the lifetime radiation exposure there would have been $10 \mathrm{mGy}$ on average, thus posing no significant risk to health.

Potential participants were identified using the data-set of a previous open questionnaire study conducted by the local governments of Nagasaki City and Nagasaki Prefecture in $2000,{ }^{16}$ which investigated health complaints among people who had lived in the relevant areas at the time of the atomic bomb attack. From among 9115 eligible inhabitants, 1614 did not respond, and we excluded 431 individuals over 80 years old owing to predicted difficulty of recruitment. The remaining sample $(n=7070)$ was stratified for gender, age group $(0-10$ years, 11-20 years and 21-25 years at the time of the bombing) and five grades of intensity of the atomic flash, heat rays and blast winds, calculated from the previous data-set, and also 
according to whether the person lived inside or outside the city area, reflecting the corresponding difference in the administrative and welfare systems. The number of individuals to be randomly selected from each cell was determined to reflect the overall distribution across all the cells, which necessitated extracting at least 406 candidates.

The control group was selected from a population who had migrated into the specified areas from outside Nagasaki City and Nagasaki Prefecture between 1950 and 1960, and thus had not experienced the explosion or had been influenced by it only negligibly. Migrants to the area during the 5 years immediately following the atomic bombing were excluded, as most of them were people returning from overseas battlefields or collapsed former Japanese colonies, who would have been severely distressed. The controls were matched with the sample group for age group, gender and current residential district, so that they would have shared subsequent life events in the community.

\section{Study design}

This was a non-blind, comparative observational study with stratified randomised sampling from the affected population with matched controls. Interviews took place during the period 12-30 March 2001. The recruitment was assisted by the Nagasaki local governments. To minimise any rater bias due to personal sympathy for atomic bomb victims, 42 psychiatrists, psychologists and social workers assisting in the study were brought in from areas of Japan other than Kyushu Island (where Nagasaki is located), with the exception of one psychiatrist who lived in Fukuoka, a prefecture in Kyushu distant from Nagasaki. None of these specialists was related to atomic bomb survivors in either Nagasaki or Hiroshima, and none of them originated from those prefectures. During the study all the raters stayed at a single hotel in Nagasaki City and were prohibited from making personal contact with the local people. They were trained in the semistructured interview procedure, and every interview was audiorecorded and randomly audited by the supervisor (Y.K.) to detect any biased interview. The local media cooperated by not interviewing any of the study participants or research staff nor issuing intermediate reports about the study, except for a simple announcement of its commencement, to prevent any of the participants receiving biased information.

The ethics committee of Kohnodai district of the National Centre of Neurology and Psychiatry, Japan, approved this study in 2001. Written informed consent was obtained from all participants. We set up a counselling hotline for those who became upset by recalling the atomic bombing, but few calls were received.

\section{Assessments}

The primary outcome measure for the group comparison was the proportion of those at high risk of mental disorder according to the 28-item version of the General Health Questionnaire (GHQ-28), using a cut-off point of 5/6; this scale assesses current mental health conditions, and its international validity has been confirmed across 15 cities in various parts of the world, fortunately including Nagasaki. ${ }^{17}$ Its cut-off point of 5/6 yields $79.7 \%$ sensitivity and $79.2 \%$ specificity, being uninfluenced by age, gender or education, for any current diagnosis of depression, dysthymia, agoraphobia, panic disorder, generalised anxiety disorder, somatisation disorder, chronic fatigue or hypochondriasis. Because the participants were aged up to 80 years, raters applying this measure were permitted to read the questions aloud and to explain the meaning of words, but not to rephrase the sentences.
The other part of the interview was semi-structured, and included questions related to demographic features, life habits, past traumatic events other than the atomic bombing, general health status, harm attributable to radioactivity among relatives and general knowledge about radioactivity. We also asked the sample group about their perception of the explosion and radioactivity, and their subsequent psychological response.

Raters were allowed to exclude any participant judged to be incapable of undergoing the interview from the assessment, based on the result of the digit span test that measures short-term memory, our own checklist of current health conditions and overall clinical impression.

\section{Statistical analysis}

The data were analysed using SPSS version 16.0 for Windows. Logistic regression was used to examine the effects on mental health of having been located in the area around the atomic bomb explosion and other factors that were significantly correlated with mental health risk using $t$ or $\chi^{2}$ tests. Logistic regression was also applied to the sample group data to examine factors that influenced current distress.

\section{Results}

For the sample group, 406 persons were invited to participate; 13 could not be traced, 12 refused and 34 could not complete the interview. For the control group, 571 persons were invited to participate; 61 could not be traced, 90 refused and 90 could not complete the interview. Also, 42 members of the control group were found to have visited the polluted area of Nagasaki City at the time of the atomic bombing. Data for 347 persons in the sample group and 288 in the control group were therefore included in the analysis.

Little difference in demographic features existed between the two groups, except for the loss of close relatives through the atomic bomb, which was more frequent in the sample group (Table 1). Poor knowledge of radioactivity was evident in both groups, especially with regard to the distinction between the atomic flash and radiation, as well as the existence of natural exposure, for which only $20.5 \%$ and $31.1 \%$ of the sample group and $27.1 \%$ and $25 \%$ of the control group respectively gave correct answers. Knowledge about radioactivity was no better in the sample group than in the controls, and was even worse for some items: $78.4 \%$ believed that they had been in the contaminated zone at the time of the explosion, thus contradicting the government's official survey. Among the controls, 20.8\% believed the same, reflecting the fact that they had lived in neighbouring prefectures at the time of the explosion - other people in the control group lived in more distant areas.

Although we intended that participants should be matched for gender and age, there was actually a significant difference in age between the sample and control groups due to the uneven response rate. Significant intergroup differences were also observed for years of education, loss of a spouse or close relatives due to the atomic bomb, and some misconceptions about radioactivity.

\section{Health findings}

A significant difference across groups was observed for mental health status, in terms both of those assessed as high risk (score $>5$ ) and of GHQ-28 total score. There was no significant difference with regard to the presence of physical disease diagnosed during the previous 6 months. 


\begin{tabular}{|c|c|c|c|c|}
\hline & $\begin{array}{l}\text { Sample group } \\
\qquad(n=347)\end{array}$ & $\begin{array}{l}\text { Control group } \\
\quad(n=288)\end{array}$ & $P$ & $\begin{array}{l}\text { Both groups } \\
\text { High } v \text {. low risk }\end{array}$ \\
\hline \multicolumn{5}{|l|}{ Demographic characteristics } \\
\hline Age, years: mean (s.d.) $)^{a}$ & $66.3(6.7)$ & $70.3(6.4)$ & $<0.001$ & $<0.01$ \\
\hline Gender: male, $n(\%)^{\mathrm{b}}$ & $131(37.8)$ & $104(36.1)$ & NS & NS \\
\hline Smoking (yes), $n(\%)^{\mathrm{b}}$ & $54(15.6)$ & $42(14.6)$ & NS & NS \\
\hline Drinking (yes), $n(\%)^{\mathrm{b}}$ & $147(42.4)$ & $116(40.3)$ & NS & NS \\
\hline Years of education: mean (s.d.) ${ }^{a}$ & $9.4(2.4)$ & $10.0(2.5)$ & $<0.001$ & $<0.05$ \\
\hline \multicolumn{5}{|l|}{ Employment history, $n(\%)^{\mathrm{b}}$} \\
\hline Office worker/civil servant & $91(26.2)$ & $84(29.2)$ & NS & NS \\
\hline Farmer/fishery & $132(38.0)$ & $102(35.4)$ & NS & $<0.05$ \\
\hline Industry worker & $42(12.1)$ & $37(12.8)$ & NS & NS \\
\hline Self-employed & $59(17.0)$ & $39(13.5)$ & NS & NS \\
\hline Other & $22(6.3)$ & $30(10.4)$ & NS & NS \\
\hline Never employed & $39(11.2)$ & $26(9.0)$ & NS & NS \\
\hline Number of family members lived with: mean (s.d. $)^{a}$ & $3.2(1.7)$ & $3.6(2.1)$ & NS & $<0.05$ \\
\hline Number of non-atomic traumatic events: mean (s.d.) ${ }^{a}$ & $4.0(2.1)$ & $4.1(2.0)$ & NS & $<0.01$ \\
\hline \multicolumn{5}{|l|}{ Loss of a spouse or up to third-degree relative } \\
\hline \multicolumn{5}{|l|}{ Erroneous knowledge about radiation, $n(\%)^{\mathrm{b}, \mathrm{c}}$} \\
\hline Radioactivity is different from flash of explosion & $276(79.5)$ & $210(72.9)$ & $<0.05$ & $<0.01$ \\
\hline Radioactivity decreases with distance & $133(38.3)$ & 116 (40.3) & NS & NS \\
\hline Radioactivity decreases with time & $172(49.6)$ & $118(41.0)$ & $<0.05$ & $<0.01$ \\
\hline Natural exposure to radiation & $239(68.9)$ & $216(75.0)$ & NS & $<0.01$ \\
\hline X-ray examination includes exposure & $134(38.6)$ & 131 (45.5) & NS & NS \\
\hline There are direct and indirect radiation effects & $88(25.4)$ & $99(34.4)$ & NS & NS \\
\hline Being exposed to bomb radiation & $272(78.4)$ & $60(20.8)$ & $<0.001$ & $<0.001$ \\
\hline \multicolumn{5}{|l|}{ Physical health finding } \\
\hline Physical disease in past 6 months, $n(\%)^{b}$ & $267(76.9)$ & $223(77.4)$ & NS & $<0.01$ \\
\hline \multicolumn{5}{|l|}{ Mental health findings (GHQ-28 score) } \\
\hline High risk, $n(\%)$ b,d & $255(73.5)$ & $114(39.6)$ & $<0.001$ & \\
\hline Total score: mean (s.d.) $)^{a}$ & $10.6(5.7)$ & $6.5(5.4)$ & $<0.001$ & \\
\hline Physical & $4.2(2.0)$ & $2.4(2.0)$ & $<0.001$ & \\
\hline Social & $1.9(1.8)$ & $1.1(1.4)$ & $<0.001$ & \\
\hline Depressive & $1.0(1.6)$ & $0.6(1.5)$ & $<0.001$ & \\
\hline Anxious & $3.5(2.0)$ & $2.3(1.8)$ & $<0.001$ & \\
\hline $\begin{array}{l}\text { GHQ-28, } 28 \text {-item General Health Questionnaire; NS, not significant. } \\
\text { a. } t \text {-test. } \\
\text { b. } \chi^{2} \text { analysis. } \\
\text { c. Erroneous } 1 \text {, correct } 0 \text {. } \\
\text { d. Cut-off score } 5 / 6 \text {. }\end{array}$ & & & & \\
\hline
\end{tabular}

\section{Correlations}

As shown in Table $1, t$-test and $\chi^{2}$ analysis revealed that poor mental health (GHQ-28 score $>5$ ) was correlated with certain variables among participants in both groups. Presence of physical illness, increased age, fewer years of education, fewer family members living together and number of traumatic life events were common stress-related factors, whereas loss of a spouse or of third-degree or closer relatives, erroneous knowledge about radiation and conviction of being contaminated by the atomic bomb explosion were specific to this research topic. Being involved in farming or fisheries was correlated with poorer mental health, presumably because of fear over soil or seawater contamination.

\section{Regression analysis}

Regression analysis was performed for both groups to clarify whether belonging to the sample group contributed to poorer mental health (Table 2). We subjected variables that contributed significantly to poorer mental health to $t$-test or $\chi^{2}$ analysis and found that group membership showed the largest contribution $(\mathrm{OR}=5.26,95 \%$ CI 2.56-11.11), whereas erroneous conceptions about radiation issues such as disagreeing with the statements 'radioactivity is not synonymous with the atomic flash' $(\mathrm{OR}=2.14,95 \% \mathrm{CI} 1.05-4.33)$ and 'natural background radiation is a normal phenomenon' $(\mathrm{OR}=2.37,95 \% \mathrm{CI} 1.16-4.84)$ also showed a modest contribution. We then performed logistic regression analysis for the sample group alone to determine which aspects of the atomic bomb experience were related to current poor mental health (Table 3); these included extent of the perceived intensity of the explosion, anxiety immediately after the explosion and anxiety on discovery of the possibility of exposure to radiological hazard. We also subjected variables that contributed significantly to poorer mental health to $t$-test or $\chi^{2}$ analysis: this revealed that only anxiety on discovery of possible radiological hazard made a significant modest contribution $(\mathrm{OR}=2.62,95 \%$ CI 1.07-6.41).

\section{Discussion}

Compared with a control group that was more rigorously selected than others used in previous studies of the effects of radiation on health, significant mental health deterioration in the absence of any proven radiation was observed in the sample group in comparison with controls who had moved to the same area 5-10 years after the explosion. This effect was modestly associated with erroneous knowledge of radiological issues, such as the mistaken belief that the atomic flash was synonymous with radiation, but was not affected by life events unrelated to radiation 


\begin{tabular}{|c|c|c|c|}
\hline & Adjusted OR & $95 \% \mathrm{Cl}$ & $\mathrm{P}$ \\
\hline Sample group ${ }^{\mathrm{b}}$ & 5.26 & $2.56-11.11$ & $<0.001$ \\
\hline Age & 0.98 & $0.93-1.04$ & NS \\
\hline Number of family members lived with & 0.91 & $0.76-1.10$ & NS \\
\hline Years of education & 1.06 & $0.90-1.24$ & NS \\
\hline Job history of farmer/fishery & 2.11 & $0.95-4.66$ & NS \\
\hline Loss of a spouse or up to third-degree relative due to atomic bomb ${ }^{b}$ & 1.75 & $0.89-3.44$ & NS \\
\hline Presence of physical disease in past 6 months ${ }^{b}$ & 1.77 & $0.82-3.80$ & NS \\
\hline Number of non-atomic traumatic events & 1.18 & $0.99-1.41$ & NS \\
\hline \multicolumn{4}{|l|}{ Erroneous knowledge of radiation ${ }^{c}$} \\
\hline Radioactivity is different from flash of explosion & 2.14 & $1.05-4.33$ & $<0.05$ \\
\hline Radioactivity decreases with time & 1.94 & $0.85-4.41$ & NS \\
\hline There is natural background exposure & 2.37 & $1.16-4.84$ & $<0.05$ \\
\hline
\end{tabular}

issues, life habits or demographic variables. Among those in the sample group, current poorer mental health was modestly associated with anxiety on learning of possible radiological harm, but not with the perceived intensity of the explosion or the subsequent anxiety it caused.

\section{Limitations}

As most measurements were based upon interview, inherent biases should be considered. A bias toward overreporting symptoms among the sample group cannot be excluded, although its effect was negligible; no significant intergroup difference in physical symptoms was observed, despite concern among the sample group about exposure to radiation and subsequent physical harm; their education, job history and life habits do not signify escapism, which was mentioned in a report from Chernobyl as a tendency for overreporting mental symptoms. ${ }^{1}$ Rater bias based on sympathy for the sample group may have led to overestimation of their sorrow and distress, as the atomic bombing is a widely recognised national tragedy and the interview was not masked; its effects were minimised by recruiting interviewers from among those who had no familial or geographical association with the atomic bomb explosion or its victims, using a semi-structured interview and randomly auditing one in ten interviews for biased questioning, although none was detected. Recall bias is inevitable in any study based on past events in that ideas about the past may be a consequence of current distress, but it would not have affected the major finding of this study, in which the primary outcome measure was current distress regarded as a variable dependent upon the group division determined by geographical location at the time of the atomic bombing.

The study design also had limitations. As it was crosssectional, it was uncertain whether the level of distress had remained the same throughout the decades since the atomic bombing, or whether it had been affected by various forms of post hoc information or social events that we did not assess. It is unlikely, however, that the association between current distress and the experience of having been located in the vicinity of the explosion, as well as learning about the potential radiological hazard, could be disproved, given the sizeable odds ratio. Furthermore, survivor bias, resulting from earlier death of more highly distressed individuals, could not be excluded. The controls would have experienced fear of soil contamination and thus might have been in poorer mental health than the general Japanese

\begin{tabular}{|c|c|c|c|}
\hline & Adjusted OR & $95 \% \mathrm{Cl}$ & $P$ \\
\hline Intensity of the the explosion perception & 1.08 & $0.82-1.42$ & NS \\
\hline Anxiety after the explosion & 0.77 & $0.36-1.64$ & NS \\
\hline Anxiety at knowing of radiological hazard & 2.62 & $1.07-6.41$ & $<0.05$ \\
\hline Age & 0.89 & $0.65-1.23$ & NS \\
\hline Number of family members lived with & 0.81 & $0.33-2.03$ & NS \\
\hline Years of education & 0.58 & $0.23-1.44$ & NS \\
\hline Loss of a spouse or up to third-degree relative due to atomic bomb ${ }^{b}$ & 3.34 & $0.53-21.07$ & NS \\
\hline Presence of physical disease in past 6 months ${ }^{b}$ & 0.87 & $0.09-8.41$ & NS \\
\hline Number of non-atomic traumatic experiences & 1.38 & $0.85-2.24$ & NS \\
\hline \multicolumn{4}{|l|}{ Erroneous knowledge of radiation ${ }^{c}$} \\
\hline Radioactivity is different from flash of explosion & 0.01 & $0.00-1.70$ & NS \\
\hline Radioactivity decreases with distance & 0.20 & $0.01-2.96$ & NS \\
\hline Natural exposure to radiation & 0.17 & $0.01-2.65$ & NS \\
\hline \multicolumn{4}{|l|}{$\begin{array}{l}\text { Model fitness } P<0.01, R^{2}=0.604 \text {, variance inflation factor } 1.101 .24 \text {. } \\
\text { NS, not significant. } \\
\text { a. High risk on the } 28 \text {-item General Health Questionnaire, cut-off score } 5 / 6 \text {. } \\
\text { b. Yes } 1 \text {, no } 0 \text {. } \\
\text { c. Erroneous } 1 \text {, correct } 0 \text {. }\end{array}$} \\
\hline
\end{tabular}


population, for whom normative data for age group are not available. However, all these factors would have reduced the extent of any intergroup difference in mental health status and would not have undermined our findings.

Within the context of these limitations, it seems reasonable to maintain the validity of the major finding that having been located within the vicinity of the atomic bomb explosion was related to prolonged mental health problems.

\section{Implications}

To our knowledge, this is the first study to have focused upon the mental health effects of psychological exposure to nuclear disaster, without radiological exposure. This issue has been touched on in earlier studies, but the findings were contaminated by the effects of radiological exposure or obscured by poorly designed controls. The observation period we employed is far longer than that of any previous studies, including those conducted after the Chernobyl nuclear accident in 1986.

Among the sample group, current poorer mental health was modestly related to anxiety upon learning about the possible risk of radiological harm, but not to the perceived intensity of the explosion or the subsequent anxiety it caused. This illustrates the importance of the adverse role of post hoc negative information, which accords with a previous report on the Three Mile Island accident in which perception of the potential harm, rather than the event itself, was related to increased distress. ${ }^{18}$ The term 'psychological fallout' could be referred to in a similar context. ${ }^{19}$ It should be noted that over half a century after the atomic bombing, three-quarters of the sample group still possessed an erroneous belief that the flash of the atomic explosion was synonymous with radioactivity. As the degree of misconception was not correlated with current mental health, the true extent and ramifications of the effect of such ignorance on persistent distress are unknown. It seems not unreasonable to speculate that many people in the sample group had failed to gain adequate access to official or medical sources of information or education, as they had not been officially registered as being exposed to radiation, despite efforts by the Nagasaki local governments to support such unregistered inhabitants.

The invisible and penetrating nature of radiation makes it difficult to define a border of safety, especially in terms of longterm harm to health, which shows a stochastic tendency; ${ }^{20}$ it is manifested only through an increased prevalence of malignancy or other diseases without any known threshold or unusual symptoms. A prevailing opinion such as that reflected in the recommendation from the International Commission on Radiological Protection in 1990 'to take every possible protective measure on the assumption that a very low dose of radiation can cause harm' would be best interpreted as a warning of future harm, ${ }^{21}$ but care should be taken to avoid causing excessive anxiety about insidious harm from past nuclear disasters, especially when associated with ambiguity or doubt about what constitutes a substantial degree of exposure.

Our study highlights a dimension of anxiety that is independent of radiological exposure, and which requires appropriate attention and treatment, or could otherwise persist over decades. An effective countermeasure might be to convert invisible harm or contamination into more tangible parameters, preferably in the acute phase, and to indicate their precise meaning. Public health promotion based on adequate information provision and psychoeducation beyond the boundary of contamination would also be important. Services based upon categorical distinction between those who are severely distressed and those who are not would create a new artificial boundary, beyond which anxiety also tends to accumulate. It is highly probable that distress of this type can affect exposed individuals as well, suggesting an explanation for the conditions known as 'atomic bomb neurosis' or 'Chernobyl syndrome', both characterised by fatigue, anxiety, depression and medically unexplained physical symptoms. ${ }^{9,22,23}$ It is noteworthy overall that sustained false apprehension about radiological harm was also related to long-term mental health deterioration. More precise details of the pathway leading from negative appraisal to poorer mental health should be clarified.

In response to our findings the Japanese government has made available financial aid for individual mental healthcare and begun a campaign of public mental health promotion for individuals who were located in the unregistered area at the time of the atomic bomb explosion. This scheme for care provision has already undergone several audits and corrections with reference to our study data, and was finally approved in 2008 .

The study will also provide some insight into the nature of the distress related to the nuclear power plant accident that occurred on 11 March 2011 in Fukushima, Japan, as a result of the great eastern Japan earthquake and subsequent tsunami. Beyond the officially recognised contaminated areas, to which entrance was still prohibited at the time of writing, low-level radioactive substances were released over a wide area. Although experts agree that the effects of this low-level radiation pose only negligible risks to health, considerable distress and anticipatory anxiety has occurred among residents in the affected areas. This situation resembles that investigated in the study reported here, and the authors (including Y.K. acting as a governmental supervisor for provision of post-disaster mental healthcare) anticipate that its findings will be useful for helping devise effective countermeasures.

\footnotetext{
Yoshiharu Kim, MD, PhD, Atsuro Tsutsumi, PhD, Takashi Izutsu, PhD, Nationa Centre of Neurology and Psychiatry; Noriyuki Kawamura, MD, PhD, Gaien Mental Clinic; Takao Miyazaki, PhD, Niigata Seiryo University Junior College; Takehiko Kikkawa, MD, PhD, National Centre of Neurology and Psychiatry and Chubu Gakuin
kina University, Tokyo, Japan

Correspondence: Dr Yoshiharu Kim, Department of Adult Mental Health, National Institute of Mental Health, National Centre of Neurology and Psychiatry, 1-4-4 Ogawa Higashi Cho, Kodaira, Tokyo 187-8553, Japan. Email: kim@ncnp.go.jp

First received 12 Oct 2010, final revision 12 May 2011, accepted 16 Jun 2011
}

\section{Funding}

The study was funded by the Ministry of Health, Labour and Welfare, Japan.

\section{Acknowledgements}

We appreciate the kind cooperation of the study participants and the local governments of Nagasaki. We also thank Dr Assen Jablenski, Dr Noriyuki Takei and Dr Sarb Johal for their kind advice, and Dr David Douglas and Maiko Fukasawa for their dedicated assistance.

\section{References}

1 United Nations Scientific Committee on the Effects of Atomic Radiation. Exposures and Effects of the Chernobyl Accident. UN, 2000.

2 World Health Organization. Chernobyl: Assessment of Radiological and Health Impacts. WHO, 2002.

3 Chernobyl Forum. Chernobyl's Legacy: Health, Environmental and SocioEconomic Impacts and Recommendations to the Governments of Belarus, the Russian Federation and Ukraine. Chernobyl Forum, 2005.

4 World Health Organization. Health Effects of the Chernobyl Accident and Special Health Care Programmes. WHO, 2006.

5 Yamada M, Kodama K, Wong FL. The long-term psychological sequelae of atomic-bomb survivors in Hiroshima and Nagasaki. In The Medical Basis 
for Radiation-Accident Preparedness (eds RC Ricks, ME Berger, FM O'Hara): 155-63. Elsevier, 1990.

6 Honda S, Shibata Y, Mine M, Imamura Y, Tagawa M, Nakane Y, et al. Mental health conditions among atomic bomb survivors in Nagasaki. Psychiatry Clin Neurosci 2002; 56: 575-83.

7 Havenaar JM, Poelijoe NW, Kasyanenko AP, Van den Bout J, Koeter MW, Filipenko VV. Screening for psychiatric disorders in an area affected by the Chernobyl disaster: the reliability and validity of three psychiatric screening questionnaires in Belarus. Psychol Med 1996; 26: 837-44.

8 Havenaar JM, Rumyantzeva GM, van den Brink W, Poelijoe NW, van den Bout $\mathrm{J}$, van Engeland $\mathrm{H}$, et al. Long-term mental health effects of the Chernobyl disaster. Am J Psychiatry 1997; 154: 1605-7.

9 Havenaar JM, de Wilde EJ, van den Bout J, Drottz-Sjoberg BM, van den Brink W. Perception of risk and subjective health among victims of the Chernobyl disaster. Soc Sci Med 2003; 56: 569-72.

10 Rahu K, Rahu M, Tekkel M, Bromet E. Suicide risk among Chernobyl cleanup workers in Estonia still increased: an updated cohort study. Ann Epidemiol 2006; 16: 917-9.

11 Loganovsky K, Havenaar JM, Tintle NL, Guey LT, Kotov R, Bromet EJ. The mental health of clean-up workers 18 years after the Chernobyl accident. Psychol Med 2008; 38: 481-8.

12 Tonnessen A, Mardberg B, Weisaeth L. Silent disaster: a European perspective on threat perception from Chernobyl far field fallout. J Trauma Stress 2002; 15: 453-9.

13 Fujita S, Kato H, Schull WJ. The LD50 associated with exposure to the atomic bombing of Hiroshima and Nagasaki. J Radiat Res (Tokyo) 1991; 32 (suppl): 154-61.
14 Houts PSC, Cleary PD, Hu TW. Distress and mediators of distress among persons living in the vicinity of Three Mile Island. In The Three Mile Island Crisis: Psychological, Social, and Economic Impacts on the Surrounding Population: 47-77. Pennsylvania State University Press, 1988.

15 Baum A, Gatchel RJ, Schaeffer MA. Emotional, behavioral, and physiological effects of chronic stress at Three Mile Island. J Consult Clin Psychol 1983; 51: 565-72.

16 Nagasaki City Citizen Station for Atomic Bomb Survivors. Report on the Testimony of the Atomic Bomb Survivors in the Unregistered Area [in Japanese]. Nagasaki City, 2000.

17 Goldberg DP, Gater R, Sartorius N, Ustun TB, Piccinelli M, Gureje O, et al. The validity of two versions of the GHQ in the WHO study of mental illness in general health care. Psychol Med 1997; 27: 191-7.

18 Goldsteen R, Schorr JK, Goldsteen KS. Longitudinal study of appraisal at Three Mile Island: implications for life event research. Soc Sci Med 1989; 28 389-98.

19 Stiehm ER. The psychologic fallout from Chernobyl. Am J Dis Child 1992; 146 761-2.

20 Mays CW. Stochastic and nonstochastic concepts: is revision needed? Health Phys 1988; 55: 437-41.

21 International Commission on Radiological Protection. 1990 Recommendations of the International Commission on Radiological Protection: 21. ICRP, 1991.

22 Lifton R. Death in Life: Survivors of Hiroshima. Random House, 1967.

23 Bromet EJ, Gluzman S, Schwartz JE, Goldgaber D. Somatic symptoms in women 11 years after the Chernobyl accident: prevalence and risk factors. Environ Health Perspect 2002; 110 (suppl 4): 625-9. 